\title{
Evolutionary games combining two or three pair coordinations on a square lattice
}

\author{
Balázs Király ${ }^{1}$ and György Szabó ${ }^{2}$ \\ ${ }^{1}$ Budapest University of Technology and Economics, Budafoki út 8, H-1111 Budapest, Hungary \\ ${ }^{2}$ Institute of Technical Physics and Materials Science, Centre for Energy Research, Hungarian Academy of Sciences, \\ P. O. Box 49, H-1525 Budapest, Hungary
}

(Received 2 July 2017; published 2 October 2017)

\begin{abstract}
We study multiagent logit-rule-driven evolutionary games on a square lattice whose pair interactions are composed of a maximal number of nonoverlapping elementary coordination games describing Ising-type interactions between just two of the available strategies. Using Monte Carlo simulations we investigate the macroscopic noise-level-dependent behavior of the two- and three-pair games and the critical properties of the continuous phase transtitions these systems exhibit. The four-strategy game is shown to be equivalent to a system that consists of two independent and identical Ising models.
\end{abstract}

DOI: 10.1103/PhysRevE.96.042101

\section{INTRODUCTION}

Due to their ubiquity in nature and society, cooperation and coordination have garnered scientific interest across various fields of research, ranging from magnetism to biology to sociophysics [1-9]. Multiagent evolutionary games [10-14] are a family of model systems used to study the spontaneous emergence of cooperation. In these models, individual players, who are located at the sites of a lattice or a graph, repeatedly play two-player games with their neighbors and change their strategies according to a dynamical rule based on their payoffs.

The concept of matrix decomposition [12,15-18] has revealed that payoff matrices, which define player incomes in two-player games $[11,12,19,20]$, are linear combinations of basis matrices that form four orthogonal subsets and represent different types of interactions: self-dependence, cross-dependence, coordination, and cyclic dominance. These game classes are clearly distinguishable via a few key matrix properties. Coordination and cyclic dominance games are defined by symmetric and antisymmetric payoff matrices whose each row and column sum is zero. A game's selfdependent components have the same entries along their rows which are equal to the row averages of the game in question. Cross-dependent components are similarly derived from column averages. This decomposition method allows us to systematically analyze how changing the payoff parameters impacts the behavior of a multiagent game system and identify those where coordination between selfish individual players may spontaneously emerge.

In the original coordination game [20-24], two players independently choose one of two available strategies. If they have both made the same choice, then they both receive 1 (unit of payoff), and they each lose the same amount otherwise. A set of $n(n-1) / 2$ similar games spans the subspace of $n$-strategy coordination-type games [16]. In these elementary coordination games, one of the $2 \times 2$ subgames (e.g., where players are restricted to use only their $i$ th and $j$ th strategies) is the aforementioned original coordination game and other strategy pairings provide no payoffs. Even among these very simple coordination-type games coordination can emerge in different ways. If strategy updates are governed by the logit rule [25-29] and the players are located at the sites of a square lattice, then the number of neutral strategies plays a decisive role [30]: When the number of strategies is below a threshold value $\left(n<n_{\text {th }}\right)$, the system undergoes a continuous (Ising-type) order-disorder phase transition as the noise level is increased; otherwise, a first-order phase transition can be observed. Adding a self-dependent component to the elementary coordination game does not necessarily destroy coordination [31]. In fact, it can be used to change the critical noise level and even the order of the phase transition.

In this paper we will continue the systematic analysis of coordination games played on a square lattice and investigate games composed of a maximal number of nonoverlapping elementary coordination games. Two elementary coordination games overlap if they share one of their coordinated strategies. It will be shown that the four- and six-strategy games are characterized by multidimensional order parameters, the phase transitions of which are still continuous; one of the fourstrategy game's phase transitions seems to still belong to the Ising class, whereas the six-strategy case is described by a different set of critical exponents. Another well-known family of coordination-type games shows a similar variety in critical behavior: Potts-type models, which are made up of all possible elementary coordination games (up to an additional irrelevant game component), also have their own universality classes [32].

The linear decomposition of payoff matrices does not only provide a systematic way to investigate the breadth of emergent phenomena associated with the family of classical spin models, but it could also similarily serve as a framework for the classification of the universality classes of critical systems. For example, Monte Carlo simulations have indicated that extending the Ising model with a sufficiently small number of neutral strategies and a properly chosen additional selfdependent game component does not change the system's critical properties $[30,31]$. Combining more elementary coordination games, however, results in models like clock models, Potts models, the Ashkin-Teller model, etc., which (with the notable exception of the two-Ising-pair game or four-state clock model $[33,34]$ ) belong to different universality classes. This suggests that a better understanding of general coordination games could lead to a deeper explanation of the concept of universality.

The structure of the rest of the paper is as follows: Section II is a detailed introduction of the models we studied. In Sec. III 
we give an overview of the methods we used to analyze these models and present our results. Section IV summarizes our main conclusions.

\section{MODELS AND GENERAL FEATURES}

We study evolutionary games on a square lattice of $N=$ $L \times L$ sites with periodic boundary conditions [11,12,14]. Each of the sites hosts one of the $N$ equivalent players who repeatedly play the same one-shot game with their four nearest neighbors. Players can choose from $n$ different pure strategies, which are represented by $n$-dimensional standard unit vectors, that is, the $i$ th strategy is represented by the $i$ th unit vector. As a shorthand we name each player after the site he or she lives on, for example, the player at site $x$ will be referred to as player $x$. Player $x$ 's income in a single round of the game is given by

$$
\tilde{u}_{x}\left(\mathbf{s}_{x}\right)=\sum_{\delta} \mathbf{s}_{x} \cdot \mathbf{A} \mathbf{s}_{x+\delta}
$$

where $\mathbf{s}_{x}$ denotes the strategy chosen by player $x$, the summation runs over their nearest neighbors at sites $x+\delta$, and the payoffs of the two-player game are defined by the $n \times n$ matrix A $[11,12,19,20]$.

Between each round of playing one-shot games, players are allowed to change their strategy in random sequential order following the so-called logit rule: The probability of player $x$ switching from strategy $\mathbf{s}_{x}$ to any available pure strategy $\mathbf{s}_{x}^{\prime}$ is

$$
w\left(\mathbf{s}_{x}^{\prime}\right)=\frac{e^{\tilde{u}_{x}\left(\mathbf{s}_{x}^{\prime}\right) / K}}{\sum_{\mathbf{s}_{x}} e^{\tilde{u}_{x}\left(\mathbf{s}_{x}\right) / K}} .
$$

In other words, players do not deterministically choose their best response, but they do exponentially favor strategies that promise higher payoffs, under the assumption that their neighbors do not modify their strategies [25-29]. The parameter $K$ can be thought of as a measure of noise affecting the decision-making abilities of individual players.

So-called potential games are games in which the incentives for unilateral strategy changes are described by a single function for all players, that is, games for which exists a $U$ potential function that satisfies

$$
\tilde{u}_{x}\left(\mathbf{s}_{x}\right)-\tilde{u}_{x}\left(\mathbf{s}_{x}^{\prime}\right)=U(\mathbf{s})-U\left(\mathbf{s}^{\prime}\right),
$$

where $\mathbf{s}_{y}^{\prime}=\mathbf{s}_{y}$ for all $y \neq x$ in the strategy profiles $\mathbf{s}=\left\{\mathbf{s}_{x}\right\}$ and $\mathbf{s}^{\prime}=\left\{\mathbf{s}_{x}^{\prime}\right\}$. In the present multiagent lattice game the whole system's potential is

$$
U(\mathbf{s})=\frac{1}{2} \sum_{x, \delta} \mathbf{s}_{x} \cdot \mathbf{V} \mathbf{s}_{x+\delta},
$$

where $\mathbf{V}$ is the potential matrix of the pair interaction. Methods for the evaluation of $\mathbf{V}$ (if it exists) are given in Ref. [12]. Potential games governed by the logit rule evolve into the Boltzmann distribution $[35,36]$ : The probability $p(\mathbf{s})$ of finding the system in strategy profile $\mathbf{s}$ becomes

$$
p(\mathbf{s})=\frac{e^{U(\mathbf{s}) / K}}{\sum_{\mathbf{s}^{\prime}} e^{U\left(\mathbf{s}^{\prime}\right) / K}} .
$$

This also means that multiagent logit-rule-driven evolutionary potential games essentially behave like classical spin models and can be studied using the principles and methods of statistical physics. Strategy profiles with maximal $U(\mathbf{s})$ correspond to preferred Nash-equilibria (ground states in the terminology of statistical physics).

All matrices that define potential games are linear compositions of cross-dependent, self-dependent, and coordinationtype games [12,15-17]. These game classes represent nonstrategic environmental effects, self-determination, and player-player interactions, respectively. A purely crossdependent payoff matrix has the same entries in each of its columns, which means that the row player has no influence over his or her payoff that is controlled by the player's surroundings instead. Conversely, a self-dependent game's payoff matrix is composed of rows filled with identical elements. In this situation the row player's choice of strategy decides his or her payoff regardless of what their opponents may do. Coordination-type games represent actual player-player interactions where both players equally affect the game's outcome.

A general $n$-strategy coordination-type game's payoff matrix is a linear combination of payoff matrices of elementary coordination games that describe Ising-type coordination between strategies $p$ and $q(p<q)$, while the remaining $n-2$ strategies are neutral, that is, choosing one of them always yields zero payoff for both players [16,30]. The elements of such an elementary payoff matrix are

$$
d_{i j}(p, q ; n)=\left\{\begin{aligned}
1 & \text { for } i=j=p \\
1 & \text { for } i=j=q \\
-1 & \text { for } i=p \text { and } j=q \\
-1 & \text { for } i=q \text { and } j=p \\
0 & \text { otherwise. }
\end{aligned}\right.
$$

A general $n$-strategy coordination-type game's payoff matrix is symmetric, and each of its row and column sums are zero [16]:

$$
\begin{aligned}
\mathbf{A}^{\text {(coord) }} & =\sum_{1 \leqslant p<q \leqslant n} \alpha_{p q} \mathbf{d}(p, q ; n) \\
& =\left(\begin{array}{cccc}
\beta_{1} & -\alpha_{12} & \cdots & -\alpha_{1 n} \\
-\alpha_{12} & \beta_{2} & \cdots & -\alpha_{2 n} \\
\vdots & \vdots & \ddots & \vdots \\
-\alpha_{1 n} & -\alpha_{2 n} & \cdots & \beta_{n}
\end{array}\right) .
\end{aligned}
$$

In games described by $\mathbf{A}^{\text {(coord) }}$ the maximal $A_{i j}$ value may be one of the diagonal elements $\beta_{p}=\sum_{q \neq p} \alpha_{p q}$, in which case all players follow the corresponding strategy in the preferred Nash equilibrium. If the sum of elementary interactions is dominated by an anticoordination game component, that is, $\max \left(A_{i j}\right)=-\alpha_{p q}$, then in the ground state the neighboring players should play different strategies, namely $p$ and $q$, to maximize payoffs. It is important to emphasize that this is only possible on bipartite lattices, like the square lattice in question, where twofold degenerate sublattice ordered strategy distributions exist. These anticoordinated systems can be mapped onto their coordinated counterparts (with two equivalent ordered homogeneous ground states) by exchanging the labels of anticoordinated strategy pairs on one of the sublattices.

In the following we will assume $n=2 m>2$ to be an even number of strategies and consider a highly symmetric family of coordination-type games, namely games composed 
of $m$ equally paying nonoverlapping elementary coordination games. (We say that two different elementary coordination games overlap if they share one of their coordinated strategies.) As a shorthand, we will call these games $m$-Ising-pair games. Up to a possible relabeling of strategies every unit-strength $m$-Ising-pair game's payoff matrix can be expanded in the following way:

$$
\mathbf{A}_{\mathrm{I}}^{(m)}=\sum_{\tau=1}^{m} \mathbf{d}(2 \tau-1,2 \tau ; 2 m) .
$$

In the $m=2$ case there are three equivalent two-Isingpair games, namely those defined by the following three commuting $4 \times 4$ payoff matrices:

$$
\begin{aligned}
\mathbf{d}(1,2 ; 4)+\mathbf{d}(3,4 ; 4) & =\left(\begin{array}{rrrr}
1 & -1 & 0 & 0 \\
-1 & 1 & 0 & 0 \\
0 & 0 & 1 & -1 \\
0 & 0 & -1 & 1
\end{array}\right), \\
\mathbf{d}(1,3 ; 4)+\mathbf{d}(2,4 ; 4) & =\left(\begin{array}{rrrr}
1 & 0 & -1 & 0 \\
0 & 1 & 0 & -1 \\
-1 & 0 & 1 & 0 \\
0 & -1 & 0 & 1
\end{array}\right), \\
\mathbf{d}(1,4 ; 4)+\mathbf{d}(2,3 ; 4) & =\left(\begin{array}{rrrr}
1 & 0 & 0 & -1 \\
0 & 1 & -1 & 0 \\
0 & -1 & 1 & 0 \\
-1 & 0 & 0 & 1
\end{array}\right) .
\end{aligned}
$$

The first of these payoff matrices, Eq. (9), is of the canonical form prescribed by Eq. (8). By swapping strategy labels 2 and 3 we get the matrix given by Eq. (10), while exchanging the labels of the second and fourth strategies leads to the payoff matrix of Eq. (11). The second matrix [see Eq. (10)] defines the model also known as the four-state clock model $[33,34,37]$ where the states are characterized by angles $s_{x}=\theta_{i}=\frac{2 \pi i}{n}(i=$ $1,2, \cdots, n)$ and the interactions between neighboring sites $(x$ and $y)$ are given as $\cos \left(\theta_{s_{x}}-\theta_{s_{y}}\right)$.

An interesting property of the four-state clock model, and consequently of the two-Ising-pair game, is its equivalence to a system made up of two identical independent uncoupled Ising models with dimensionless coupling coefficients of $1 / 2$ [34]. If we assume that the two-Ising-pair game has only one phase transition, then Kramers and Wannier's duality argument [38-41] predicts that its critical temperature is

$$
K_{\mathrm{c}}(2)=\frac{1}{\ln (\sqrt{2}+1)} \simeq 1.134593 .
$$

This is the half of the critical temperature $K_{\mathrm{c}}(1)$ of the traditional Ising model [42], and in the present notation $K_{\mathrm{c}}(1)=2 K_{\mathrm{c}}(2)$.

This equivalence can actually be considered as a special case of a correspondence between two more general models that are also related to two-Ising-pair games: the Ashkin-Teller model and a two-layer Ising model with four-spin interactions.

The Ashkin-Teller model [43] is a four-state model in which the pair interactions are defined by the following payoff matrix:

$$
\mathbf{A}=\left(\begin{array}{cccc}
\varepsilon & \varepsilon^{\prime} & \varepsilon^{\prime \prime} & \varepsilon^{\prime \prime \prime} \\
\varepsilon^{\prime} & \varepsilon & \varepsilon^{\prime \prime \prime} & \varepsilon^{\prime \prime} \\
\varepsilon^{\prime \prime} & \varepsilon^{\prime \prime \prime} & \varepsilon & \varepsilon^{\prime} \\
\varepsilon^{\prime \prime \prime} & \varepsilon^{\prime \prime} & \varepsilon^{\prime} & \varepsilon
\end{array}\right) .
$$

In the terminology of game theory this interaction is composed of the three games defined by Eqs. (9)-(11) with coefficients $\left(\varepsilon^{\prime}-\bar{\varepsilon}\right),\left(\varepsilon^{\prime \prime}-\bar{\varepsilon}\right)$, and $\left(\varepsilon^{\prime \prime \prime}-\bar{\varepsilon}\right)$, where $\bar{\varepsilon}=\left(\varepsilon+\varepsilon^{\prime}+\varepsilon^{\prime \prime}+\right.$ $\left.\varepsilon^{\prime \prime \prime}\right) / 4$, and an irrelevant component described by the all-one matrix. Evidently, this system becomes equivalent to the traditional Potts model [33] if $\varepsilon^{\prime}=\varepsilon^{\prime \prime}=\varepsilon^{\prime \prime \prime}=-\varepsilon / 3=-1$.

The states of the Ashkin-Teller model can alternatively be represented by introducing two Ising spins $\left(S_{x}, T_{x}= \pm 1\right)$ at each site of the lattice and assigning the two-spin states $(+,+),(+,-),(-,+)$, and $(-,-)$ to strategies $1,2,3$, and 4 , respectively $[34,40,44]$. In the latter notation the pair interaction between the neighboring players $x$ and $y$ is given by the following expression:

$$
J_{0}+J^{\prime} S_{x} S_{y}+J^{\prime \prime} T_{x} T_{y}+J_{4} S_{x} S_{y} T_{x} T_{y} .
$$

where two Ising models with coupling coefficients $4 J^{\prime}=$ $\varepsilon+\varepsilon^{\prime}-\varepsilon^{\prime \prime}-\varepsilon^{\prime \prime \prime}$ and $4 J^{\prime \prime}=\varepsilon-\varepsilon^{\prime}+\varepsilon^{\prime \prime}-\varepsilon^{\prime \prime \prime}$ are coupled via four-body interactions characterized by $4 J_{4}=\varepsilon-\varepsilon^{\prime}-\varepsilon^{\prime \prime}+$ $\varepsilon^{\prime \prime \prime}$. The $J_{0}=\bar{\varepsilon}$ term simply shifts the system's ground-state energy. The symmetry of the original $\varepsilon$ parameters also implies that the model is invariant under permutations of the parameters $J^{\prime}, J^{\prime \prime}$, and $J_{4}$ [45].

Even though, at first sight, the $m$-Ising-pair coordination game might look similar to the Potts model in having $n=$ $2 m$ symmetry-related strategies (and, consequently, $n$-fold degenerate ground states), this interaction is "less symmetric" because its strategies are only pairwise interchangeable. Furthermore, the traditional $n$-strategy Potts model is the linear combination of all (and not just a few) $n$-strategy elementary coordination games with equal strengths and an irrelevant game component that shifts all payoffs by a constant value.

Finally, we mention that the equivalence between ferromagnetic and antiferromagnetic Ising models can be adapted for each coordinated strategy pair by exchanging the suitable strategy labels on one of the two equivalent sublattices $[9,46]$. This feature expands the validity region of the subsequent calculations.

\section{METHODS}

\section{A. Mean-field approximation}

The difference between the $m$-Ising-pair coordination game and the $n=2 m$-state Potts model already emerges at the level of mean-field approximations. In these calculations it is more convenient to use the scalar notation of strategies: $s_{x}=k$ with $k=1,2, \ldots, n$ when player $x$ uses her $k$ th strategy. The mean-field approximation describes the systems with the average strategy frequencies $\left(\rho_{k}\right)$. For translation invariant connectivity structures the one-site probabilities $\left[p_{1}(k)=\rho_{k}\right]$ of finding the $k$ th strategy at any one of the sites are used to express the probability of the microscopic state $\mathbf{s}$ as $p(\mathbf{s})=$ $\prod_{x} p_{1}\left(s_{x}\right)$. Detailed descriptions of this approach (as well as its generalized versions) are given in Refs. [12,47-49]. For these methods the equilibrium values of strategy frequencies (or $n$-site configuration probabilities) are determined as extremal points of a thermodynamic potential, in our case

$$
\Phi=\mathcal{U}+K \mathcal{S} .
$$

Here $\mathcal{U}$ and $\mathcal{S}$ are the average values of the potential $[\mathcal{U}=$ $\left.\sum_{\mathbf{s}} p(\mathbf{s}) U(\mathbf{s})\right]$ and the entropy $\left[\mathcal{S}=-\sum_{\mathbf{s}} p(\mathbf{s}) \ln p(\mathbf{s})\right]$ of the 


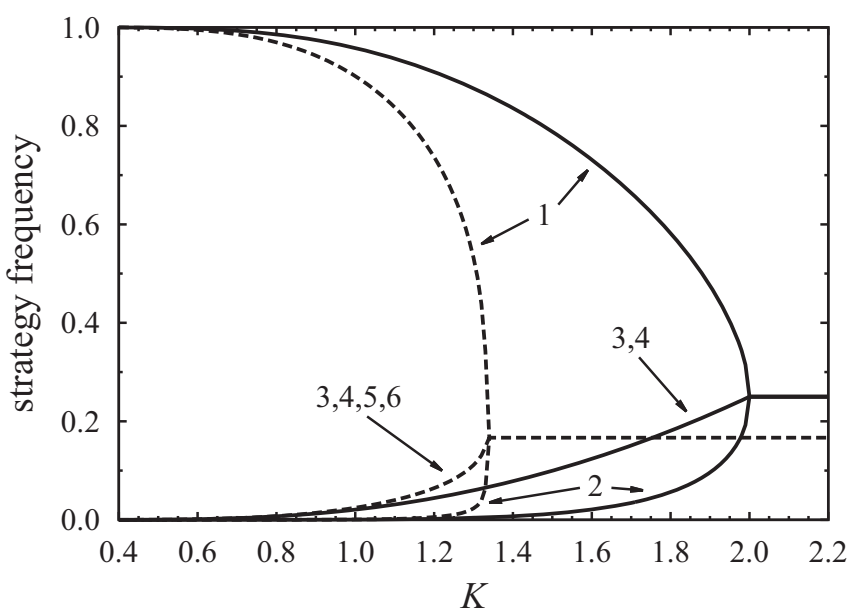

FIG. 1. Strategy frequencies as a function of the noise level $K$ in the mean-field approximation of the two- and three-pair-Ising coordination games. The solid lines correspond to the two-pair, while the dashed lines represent the three-pair case.

whole system. It should be emphasized that in game-theoretic systems payoffs, and therefore the potential, is maximal in the ground state, contrary to physical systems where the energy is minimal. This is why the thermodynamic potential $\Phi$ differs from the usual free energy in the sign of the entropic term $K \mathcal{S}$.

We have plotted the stable numerical solutions of the mean-field theory of the two- and three-Ising-pair coordination games in Fig. 1. Both plots clearly indicate the presence of a continuous (critical) phase transition at $K=K_{\mathrm{c}}$. For $K>K_{\mathrm{c}}$ the equilibrium state is disordered, and all strategies are present in the system with equal frequencies $\left(\rho_{k}=1 / n\right)$. This symmetry is spontaneously broken as the noise level is lowered below a critical value: One strategy's frequency rises (labelled as strategy 1 in Fig. 1, but we also have a similar, symmetry-related, equally preferred state for each of the remaining strategies) while the frequencies of the other

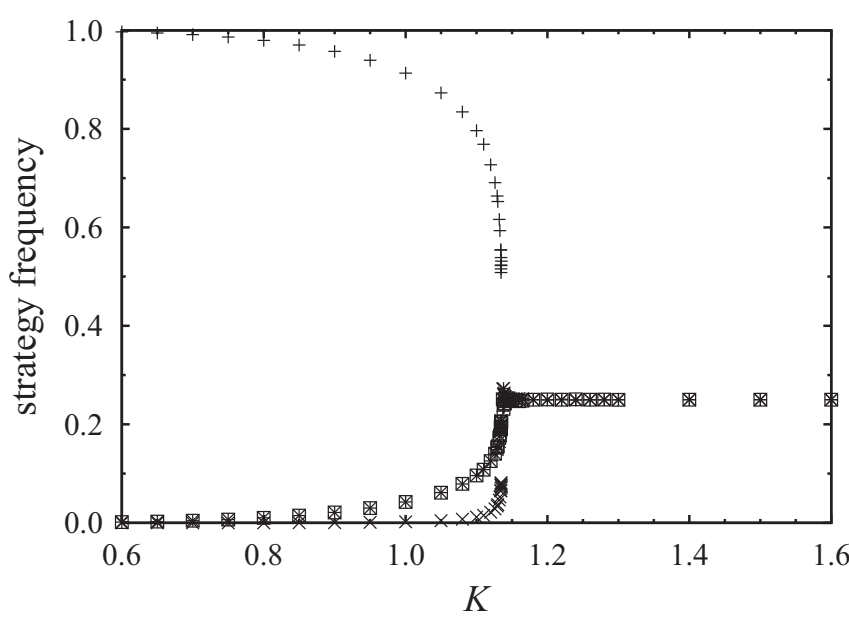

FIG. 2. Strategy frequencies plotted against the noise level $K$ in the four-strategy $(m=2)$ case. Strategies 1 and 2 (pluses and crosses) belong to the first pair while strategies 3 and 4 (stars and boxes) represent the second coordinated pair. strategies diminish. At the same time the second strategy's frequency decreases at a faster rate than the rest, while the symmetry of the other Ising pairs is retained.

At very low noise levels the system becomes homogeneously ordered. As only one Ising pair's symmetry is broken below the critical noise level, the remaining strategies are found on neighboring sites with equal probabilities. This means that at the mean-field level the expected payoff for players following these strategies becomes zero, and therefore the strategies themselves are effectively neutral. In other words, the $m$-Ising-pair game is mean-field equivalent to the $2 m$-strategy elementary coordination game, whose mean-field behavior was previously studied in Ref. [30].

We emphasize that the $m$-Ising-pair game has $2 m$ equivalent, equally preferred ordered ground states on a square lattice. Similar degeneracy occurs for the $2 m$-state Potts model that undergoes a first-order phase transition if $n=2 m>4$ [32]. In light of the mean-field results we can introduce two order parameters, namely $M_{1}(K, m)$ and $M_{2}(K, m)$. Without loss of generality henceforth we assume that $\rho_{1} \rightarrow 1$ in the low-noise limit while $\rho_{2}<\rho_{3}=\rho_{4} \rightarrow 0$. Then the order parameters can be given by the average strategy frequencies as

$$
\begin{gathered}
M_{1}(K, m)=\rho_{1}-\rho_{2}, \\
M_{2}(K, m)=1-2 m \rho_{i} \text { if } i>2 .
\end{gathered}
$$

For these choices both order parameters go to 1 in the low-noise limit and vanish in the symmetric (high-noise) states. Additionally, the mean-field analysis predicts algebraic behaviors

$$
M_{j}(K, m) \propto\left(K_{\mathrm{c}}(m)-K\right)^{\beta_{j}(m)} \text { for } j=1,2,
$$

when $K \rightarrow K_{\mathrm{c}}(m)$ from below. Figure 1 illustrates clearly that $K_{\mathrm{c}}(2)=2, \beta_{1}^{(\mathrm{mf})}(2)=1 / 2$, and $\beta_{2}^{(\mathrm{mf})}(2)=1$. For $m=3$ the numerical investigation of the mean-field solution indicates a significantly different continuous phase transition with $K_{\mathrm{c}}(3)=4 / 3, \beta_{1}(3)=1 / 4$, and $\beta_{2}(3)=1 / 2$.

\section{B. Monte Carlo simulations}

Monte Carlo (MC) simulations were also performed to investigate the features of coordination games $\mathbf{A}_{\mathrm{I}}^{(m)}$ composed of $m=2$ and 3 elementary pair coordination components. The games were studied on an $N=L \times L$-site square lattice using periodic boundary conditions in order to better approximate infinite-size thermodynamic systems. The linear size of the system was varied from $L=400$ to 2000. Average strategy frequencies $\rho_{i}=\left\langle\rho_{i}(t)\right\rangle$ were evaluated by averaging over a sampling time $t_{\mathrm{s}}$ after waiting for a relaxation time $t_{\mathrm{r}}$. Both the sampling and relaxation times were varied between $10^{3}$ and $10^{6}$ Monte Carlo steps. During a Monte Carlo step each of the players received one chance, on average, to change their strategy. The larger system sizes and the longer run times were used near the critical phase transitions to overcome the effects of diverging fluctuations, correlation lengths, and relaxation times. Furthermore, below the critical transition points $\left[K<K_{\mathrm{c}}(m)\right]$ preordered initial states (with $\rho_{1}=1$ ) were used to reduce the duration of transient phenomena. (This did not change the equilibrium state itself.) 


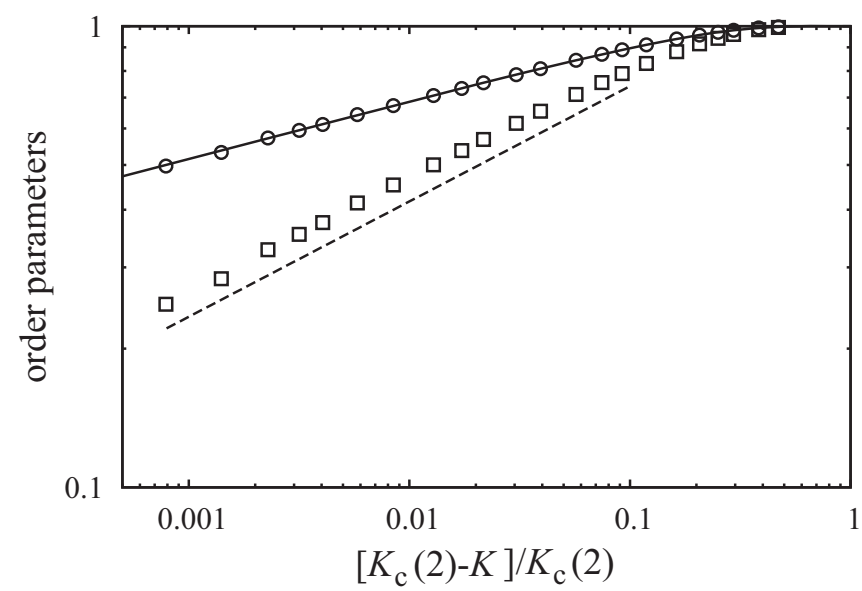

FIG. 3. Log-log plot of the order parameters versus the relative noise level $\left(K_{\mathrm{c}}(2)-K\right) / K_{\mathrm{c}}(2)$ for $m=2$. The MC data of $M_{1}(K, 2)$ and $M_{2}(K, 2)$ are denoted by open circles and squares. The solid line shows Onsager's exact result [42] for $m=1$. The dashed line (with a slope of 1/4) serves as a reference illustrating another power-law behavior.

The MC data of strategy frequencies $\rho_{i}(i=1,2,3$, and 4) (see Fig. 2) clearly show that the system undergoes a continuous phase transition at $K_{\mathrm{c}}^{(\mathrm{MC})}(2) \approx 1.135(1)$. Within our statistical error this $\mathrm{MC}$ result agrees with the theoretical prediction of Eq. (12). Above this critical temperature the system evolves into a disordered state, in which all available strategies are played with an equal probability at any given site. If $K<K_{\mathrm{c}}(2)$, then the symmetry of one of the Ising pairs is broken.

Figure 3 illustrates quantitatively the critical (algebraic) behavior of the order parameters $M_{1}(K, 2)$ and $M_{2}(K, 2)$ when $K \rightarrow K_{\mathrm{c}}(2)$ from below. We emphasize that Onsager's exact result for $M_{1}(K, 1)$ and $M_{1}(K, 2)$ coincide as functions of relative noise levels $\left(K_{\mathrm{c}}(m)-K\right) / K_{\mathrm{c}}(m)$. At the same time this means that the critical exponent of $M_{1}(K, 2)$ is $\beta_{1}^{(\mathrm{MC})}(2)=1 / 8$.

The second order parameter $M_{2}(K, 2)$ also follows a power law with a larger exponent whose value can be approximated as $\beta_{2}^{(\mathrm{MC})}(2) \simeq 1 / 4$ within our statistical error. Accordingly, both the mean-field approximation and the MC simulations predict that $\beta_{2} / \beta_{1}=2$.

The power-law decrease of order parameters is generally accompanied by the divergence of other quantities in the close vicinity of the critical point. In order to explore this aspect of the two-Ising-pair model our MC simulations were extended to quantify the fluctuations of the order parameters. More precisely, we have evaluated the following quantities:

$$
\begin{aligned}
& \chi_{1}=N\left[\left\langle\left(\rho_{1}(t)-\rho_{2}(t)\right)^{2}\right\rangle-\left(\rho_{1}-\rho_{2}\right)^{2}\right], \\
& \chi_{2}=\frac{N}{n-2} \sum_{i>2}^{n}\left\langle\left(\rho_{i}(t)-\rho_{i}\right)^{2}\right\rangle, \\
& \chi^{\prime}=\frac{N}{n} \sum_{i=1}^{n}\left\langle\left(\rho_{i}(t)-\frac{1}{n}\right)^{2}\right\rangle,
\end{aligned}
$$

where $\chi_{1}$ measure the fluctuations of order parameter $M_{1}(K, m)$ if $K<K_{\mathrm{c}}(m), \chi_{2}$ quantifies the fluctuations for the

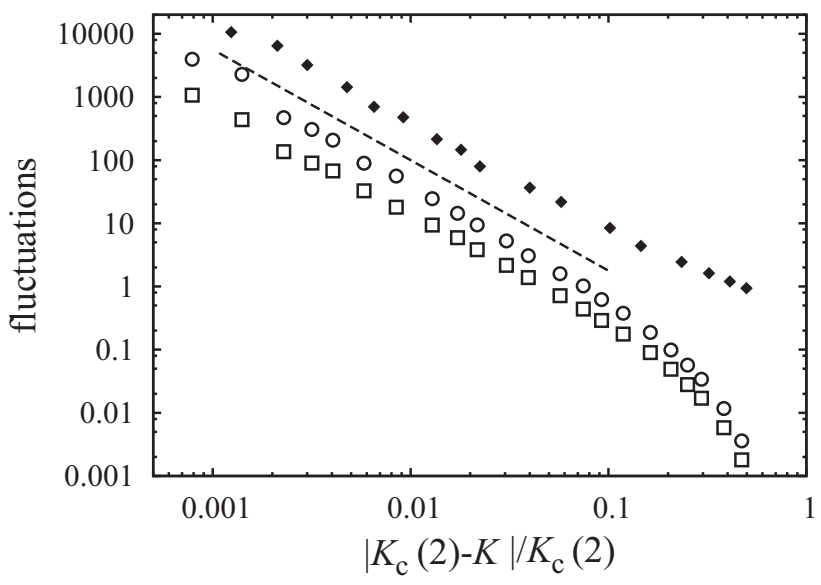

FIG. 4. Log-log plot of fluctuations as a function of $\mid K-$ $K_{\mathrm{c}}(2) \mid / K_{\mathrm{c}}(2)$ for $m=2$. Open circles and boxes show the divergence of $\chi_{1}$ and $\chi_{2}$ below the critical noise level. Black diamonds denote MC data for $\chi^{\prime}$ if $K>K_{\mathrm{c}}(2)$. The dashed line represents the trend of divergence in $\chi_{1}$ and $\chi^{\prime}$ for the two-dimensional Ising model $(m=1)$.

identical strategy frequencies $\rho_{i}\left(2<i \leqslant m, K<K_{\mathrm{c}}(m)\right)$, while $\chi^{\prime}$ quantifies the fluctuation of strategy frequencies in the disordered phase. It is worth mentioning that averaging over equivalent strategies improves statistical accuracy. Furthermore, these notations remain valid for $n=2 m>4$.

From our MC simulations (see Fig. 4) one can conclude that the above quantities diverge as $\left|K_{\mathrm{c}}(2)-K\right|^{-\gamma}$ when approaching the critical point. Within the statistical error the value of the exponent $\gamma$ agrees with those found for the twodimensional Ising model $(\gamma=7 / 4)$ [50-52]. These numerical data suggest that the robustness of the Ising universality class is not disturbed by an additional coordinated strategy pair in the corresponding four-strategy evolutionary game. Here we emphasize that similar phenomena were observed for models where the coordinated strategy pair is extended by neutral strategies [30,31].

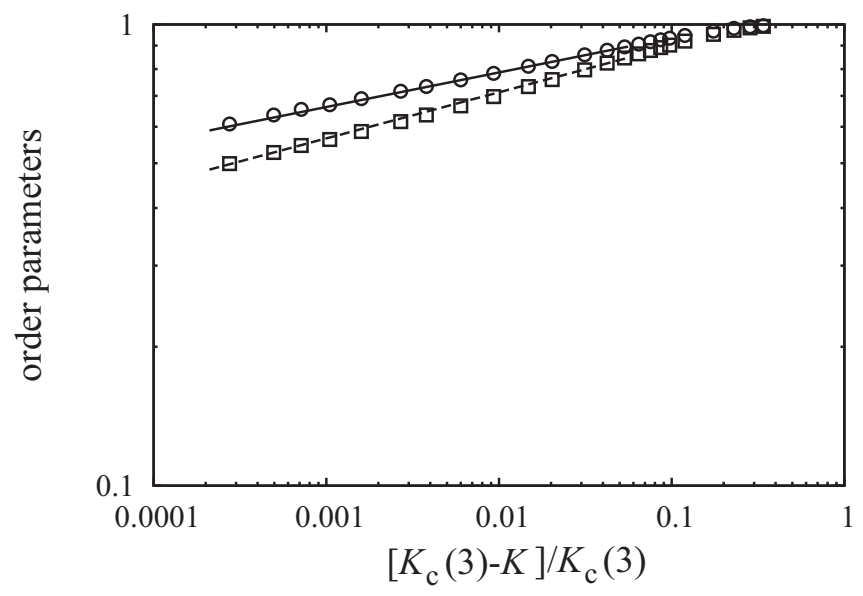

FIG. 5. Log-log plot of MC data for the order parameters $M_{1}(K, 3)$ (circles) and $M_{2}(K, 3)$ (boxes) as a function of $\left[K_{\mathrm{c}}(3)-\right.$ $K] / K_{\mathrm{c}}(3)$. The solid and dashed lines (with slopes of 0.075 and 0.1 , respectively) correspond to the fitted power laws for $M_{1}(K, 3)$ and $M_{2}(K, 3)$. 


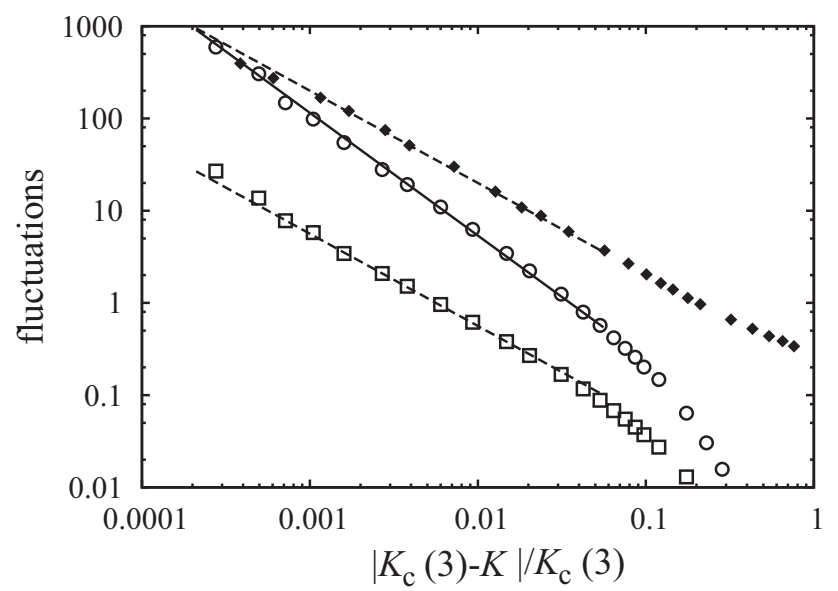

FIG. 6. Log-log plot of the MC results of $\chi_{1}(K, 3)$ (diamonds), $\chi_{2}(K, 3)$ (boxes), and $\chi^{\prime}(K, 3)$ (circles) versus the relative noise level. The solid and dashed lines represent power laws with exponents $\gamma=4 / 3$ and 1 .

Fitting the power-law assumption to the 13 data points closest to the critical temperature yields the following approximations of the critical exponents: $\beta_{1}^{(\mathrm{f})}(2)=0.124(1)$, $\beta_{2}^{(\mathrm{f})}(2)=0.247(2), \gamma_{1}^{(\mathrm{f})}(2)=1.85(4), \gamma_{2}^{(\mathrm{f})}(2)=1.69(2)$, and $\gamma^{\prime(\mathrm{f})}(2)=1.68(3)$.

A more accurate numerical analysis of the critical behavior goes beyond the scope of this work. Instead, we briefly survey the $\mathrm{MC}$ results obtained for the six-strategy model involving three nonoverlapping coordinated strategy pairs. The temperature dependence of strategy frequencies is qualitatively similar to the one obtained in the $m=2$ case. More precisely, the system exhibits a continuous phase transition which only breaks the symmetry of one of the constituent Ising pairs. In agrement with expectations, the critical temperature is lower, according to the MC simulations $K_{\mathrm{c}}^{(\mathrm{MC})}(3)=0.9084(1)$. Here the duality relations cannot be used to deduce exact analytical result. Figure 5 illustrates the power-law behavior of $M_{1}(K, 3)$ with an estimated exponent of $\beta_{1}(3)=0.075$. On the same figure $M_{2}(K, 3)$ increases with an approximated slope of $\beta_{2}(3)=0.1$.

Figure 6 illustrates the divergence of the quantities $\chi_{1}, \chi_{2}$, and $\chi^{\prime}$ in the close vicinity of the critical point for $m=3$. Contrary to the case of $m=2$, here we can predict two values for the $\gamma$ exponents: $\gamma_{1}(3) \simeq 4 / 3$ and $\gamma_{2}(3)=\gamma^{\prime}(3) \simeq 1$.

Fitting the 13 data points closest to the critical points gives the following critical exponents: $\beta_{1}^{(\mathrm{f})}(3)=0.0745(5)$, $\beta_{2}^{(\mathrm{f})}(3)=0.102(1), \gamma_{1}^{(\mathrm{f})}(3)=1.33(2), \gamma_{2}^{(\mathrm{f})}(3)=1.08(2)$, and $\gamma^{\prime(\mathrm{f})}(3)=0.93(2)$

\section{SUMMARY}

In this paper we have investigated the critical phase transitions in evolutionary potential games with $n=4$ or 6 strategies when the pair interactions are made up of two or three nonoverlapping elementary coordination games describing equivalent Ising-like interactions between the strategy pairs. The players are located at the sites of a square lattice, they interact with their four nearest neighbors, and their strategy updates are dictated by the logit rule and stationary states are described by the Boltzmann distribution [12].

These models share some important features with some traditional models, namely the ground state is $n$-fold degenerate and these systems exhibit a continuous order-disorder phase transition when the noise level is increased from zero. For $n=4$ three equivalent models can be distinguished, one of which is identical to the four-state clock model [33]. The linear combinations of these three components reproduce the set of Ashkin-Teller models [43] for which a duality relation [53,54] can be exploited to exactly determine the critical noise level [41]. Mean-field theory suggests that two order parameters can be introduced as suitable combinations of strategy frequencies. These quantities vanish algebraically when approaching the critical point from below. Using Monte Carlo simulations we have determined the corresponding critical exponents for both order parameters and also for the diverging fluctuations of strategy frequencies. The comparison of these data with the exact results of the traditional Ising model has indicated some surprising coincidences. Namely, the exact critical noise level is half of that characteristic of the Ising model [41]. Furthermore, within statistical error the plot of $\rho_{1}-\rho_{2}$ versus the relative noise level reproduces Onsager's result for the magnetization of the Ising model. This means that one of the critical exponents of the order parameters is $\beta_{1}=1 / 8$ while the other seems to be $\beta_{2}=1 / 4$. At the same time the fluctuations of the strategy frequencies diverge algebraically with the same exponent when approaching the critical point from both sides.

For the six-strategy model the continuous order-disorder phase transition can also be characterized by two order parameters. According to our Monte Carlo analysis the critical exponents are smaller than those found for $n=4$. Contrary to the previous case, the divergence of strategy frequency fluctuations at the critical point can be characterized by two different exponents.

Finally, we emphasize that all the above discussed models posses some symmetries in the structure of pair interactions (matrices) belonging to the class of coordination type games [17]. One can introduce, however, further symmetric combinations of elementary coordination games that can also result in critical phase transitions that do not belong to any of the traditional universality classes $[50,55]$. Accordingly, the possible combinations can give us a framework for the classification of universality classes.

\section{ACKNOWLEDGMENT}

This work was supported by the Hungarian National Research Fund (Grant No. OTKA K-120785).
[1] S. Galam, Y. Gefen, and Y. Shapir, Math. J. Sociol. 9, 1 (1982).

[2] A. V. M. Herz, J. Theor. Biol. 169, 65 (1994).
[3] W. A. Brock and S. N. Durlauf, Rev. Econ. Stud. 68, 235 (2001).

[4] G. Weisbuch and D. Stauffer, Physica A 384, 542 (2007). 
[5] S. Galam and B. Walliser, Physica A 389, 481 (2010).

[6] S. Grauwin, D. Hunt, E. Bertin, and P. Jensen, Adv. Complex Syst. 14, 529 (2011).

[7] S. M. Krause and S. Bornholdt, Physica A 392, 4048 (2013).

[8] J.-P. Bouchaud, J. Stat. Phys. 151, 567 (2013).

[9] D. Sornette, Rep. Prog. Phys. 77, 062001 (2014).

[10] M. A. Nowak, Evolutionary Dynamics (Harvard University Press, Cambridge, MA, 2006).

[11] G. Szabó and G. Fáth, Phys. Rep. 446, 97 (2007).

[12] G. Szabó and I. Borsos, Phys. Rep. 624, 1 (2016).

[13] C. P. Roca, J. A. Cuesta, and A. Sánchez, Phys. Life Rev. 6, 208 (2009).

[14] B. Allen and M. A. Nowak, EMS Surv. Math. Sci. 1, 113 (2014).

[15] O. Candogan, I. Menache, A. Ozdaglar, and P. A. Parrilo, Math. Oper. Res. 36, 474 (2011).

[16] G. Szabó, K. S. Bodó, B. Allen, and M. A. Nowak, Phys. Rev. E 90, 042811 (2014).

[17] G. Szabó, K. S. Bodó, B. Allen, and M. A. Nowak, Phys. Rev. E 92, 022820 (2015).

[18] G. Szabó, K. S. Bodó, and K. A. Samani, Phys. Rev. E 95, 012320 (2017).

[19] J. von Neumann and O. Morgenstern, Theory of Games and Economic Behaviour (Princeton University Press, Princeton, NJ, 1944).

[20] K. Sigmund, The Calculus of Selfishness (Princeton University Press, Princeton, NJ, 2010).

[21] R. Gibbons, Game Theory for Applied Economists (Princeton University Press, Princeton, NJ, 1992).

[22] M. J. Osborn and A. Rubinstein, A Course in Game Theory (MIT Press, Cambridge, MA, 1994).

[23] J. W. Weibull, Evolutionary Game Theory (MIT Press, Cambridge, MA, 1995).

[24] R. Cooper, Coordination Games (Cambridge University Press, Cambridge, 1998).

[25] J. S. Cramer, Logit Models from Economics and Other Fields (Cambridge University Press, Cambridge, 2003).

[26] G. W. Brown, in Activity Analysis of Production and Allocation, edited by T. C. Koopmans (Wiley, New York, 1951), pp. 373-376.
[27] D. Monderer and L. S. Shapley, J. Econ. Theory 68, 258 (1996).

[28] W. H. Sandholm, Games 1, 3 (2010).

[29] G. Szabó and A. Szolnoki, J. Theor. Biol. 299, 81 (2012).

[30] G. Szabó and B. Király, Phys. Rev. E 93, 052108 (2016).

[31] B. Király and G. Szabó, Phys. Rev. E 95, 012303 (2017).

[32] F. Y. Wu, Rev. Mod. Phys. 54, 235 (1982).

[33] R. B. Potts, Math. Proc. Camb. Phil. Soc. 48, 106 (1952).

[34] M. Suzuki, Prog. Theor. Phys. 37, 770 (1967).

[35] L. E. Blume, Games Econ. Behav. 5, 387 (1993).

[36] L. E. Blume, Games Econ. Behav. 44, 251 (2003).

[37] J. Tobochnik, Phys. Rev. B 26, 6201 (1982)

[38] H. A. Kramers and G. H. Wannier, Phys. Rev. 60, 252 (1941).

[39] H. A. Kramers and G. H. Wannier, Phys. Rev. 60, 263 (1941).

[40] C. Fan, Phys. Lett. A 39, 136 (1972).

[41] J. Chen, H.-J. Liao, H.-D. Xie, X.-J. Han, R.-Z. Huang, S. Cheng, Z.-C. Wei, Z.-Y. Xie, and T. Xiang, Chin. Phys. Lett. 34, 050503 (2017).

[42] L. Onsager, Phys. Rev. 65, 117 (1944).

[43] J. Ashkin and E. Teller, Phys. Rev. 64, 178 (1943).

[44] R. J. Baxter, Exactly Solved Models in Statistical Mechanics (Academic Press, London, 1982).

[45] F. Y. Wu and K. Y. Lin, J. Phys. C: Solid Stat. Phys. 7, L181 (1974).

[46] S. G. Brush, Rev. Mod. Phys. 39, 883 (1967).

[47] R. Kikuchi and S. Brush, J. Chem. Phys. 47, 195 (1967).

[48] T. Morita, Prog. Theor. Phys. Suppl. 115, 27 (1994).

[49] D. de Fontaine, in Theory and Applications of the Cluster Variation and Path Probability Methods, edited by J. L. MorinLopez and J. M. Sanchez (Springer, Berlin, 1996), pp. 125-144.

[50] H. E. Stanley, Introduction to Phase Transitions and Critical Phenomena (Clarendon Press, Oxford, 1971).

[51] M. Plischke and B. Bergersen, Equilibrium Statistical Physics (World Scientific, Singapore, 1996).

[52] J. Cardy, Scaling and Renormalization in Statistical Physics (Cambridge University Press, Cambridge, 1996).

[53] F. J. Wegner, J. Math. Phys. 12, 2259 (1971).

[54] F. J. Wegner, J. Phys. C: Solid Stat. Phys. 5, L131 (1972).

[55] H. E. Stanley, Rev. Mod. Phys. 71, S358 (1999). 